\title{
Measurement of Cellular Excitability by Whole Cell Patch Clamp Technique
}

\author{
M. KARMAŽÍNOVÁ ${ }^{1}$,'. LACINOVÁ ${ }^{1}$ \\ ${ }^{1}$ Slovak Academy of Sciences, Institute of Molecular Physiology and Genetics, Centre of \\ Excellence of the Slovak Research and Development Agency "Biomembranes2008" and Centre of \\ Excellence for Cardiovascular Research, Bratislava, Slovak Republic
}

Received January 28, 2010

Accepted March 26, 2010

\begin{abstract}
Summary
Patch clamp method developed more than 30 years ago is widely used for investigation of cellular excitability manifested as transmembrane ionic current and/or generation of action potentials. This technique could be applied to measurement of ionic currents flowing through individual (single) ion channels or through the whole assembly of ion channels expressed in the whole cell. Whole cell configuration is more common for measurement of ion currents and the only one enabling measurement of action potentials. This method allows detailed analysis of mechanisms and structural determinants of voltagedependent gating of ion channels as well as regulation of channel activity by intracellular signaling pathways and pharmacological agents.
\end{abstract}

\section{Key words}

Patch clamp • Calcium current • Activation • Deactivation • Inactivation

\section{Corresponding author}

L'. Lacinová, Institute of Molecular Physiology and Genetics, Slovak Academy of Sciences, Vlárska 5, 83334 Bratislava, Slovak Republic. Fax: +421-2-5477 3666. E-mail: lubica.lacinova@savba.sk

\section{Introduction}

Neher, Sackmann and collaborators (Hamill et al. 1981) developed the patch clamp method at the end of seventies - beginning of eighties of the twentieth century.
This method optimizes measurement of electric events from small cells. Principle of the method is near-perfect electrical isolation of small "patch" of cell membrane inside the tip of glass electrode, which is reached by gentle pressing of the pipette against the cell followed by gentle suction applied through the pipette. Electric resistance of the cell-pipette junction must exceed $1 \mathrm{G} \Omega$ (so-called gigaseal). Under these conditions the background leak current caused by flow of random ions between the cell surface and the surface of the pipette tip should not exceed $10 \mathrm{pA}$ so that the measurement of the current carried through the expressed ion channels is not disturbed.

Establishing of the gigaseal is a starting point for several possible patch clamp configurations: cellattached, inside-out, outside-in or whole cell (Hamill et al. 1981). First three configurations can be used for recording the activity of a single channel. The whole cell mode is used for monitoring the activity of all channels expressed in the investigated cell. This is the most common configuration used in electrophysiological experiments.

By application of additional suction membrane, the patch isolated inside the pipette tip will break and electrical contact between the cytoplasm and an electrode placed inside the pipette and filled by intracellular solution is established. In addition, intracellular solution slowly diffuses from the pipette into cytoplasm. The examined cell is placed in the experimental chamber filled with a bath solution and containing the second electrode. This electrode is grounded and keeps the 
A

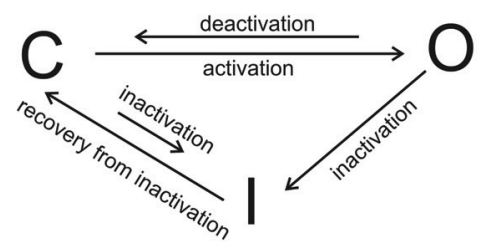

B

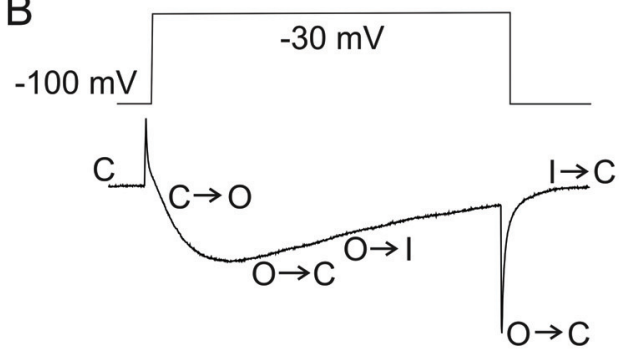

Fig. 1. Diagram of gating transitions of a voltage-dependent ion channel (A). Letters $\mathrm{C}, \mathrm{O}$ and I represent a closed or resting channel state, an open or activated channel state and an inactivated channel state, respectively. Size of arrows illustrates relative probability of individual transitions. An example of inward current recorded from T-type calcium channel during depolarizing pulse is shown in (B). electrical potential of the outer side of cell membrane at $0 \mathrm{mV}$. Electrical potential of the inner side of cell membrane is controlled through an intracellular electrode by a patch clamp amplifier.

Patch clamp technique has been successfully used for measurement of ionic flow caused by the activity of various ion transporting proteins including voltageoperated ion channels, ligand-operated ion channels and electrogenic ion exchangers. The most common application of the whole-cell patch clamp is the analysis of the function of voltage-operated ion channels.

The whole cell patch clamp can operate in two recording modes depending on the configuration of a patch clamp amplifier. In the "voltage clamp" mode the recorded signal is the transmembrane current and the controlled input is the "clamped" membrane voltage delivered to the cell examined. In the "current-clamp" mode the input is the current that is injected into the cell and changes are recorded in the membrane potential, e.g., generation of an action potential.

\section{Activity of voltage-dependent channels monitored by whole-cell patch clamp}

Excitable cells may express four types of voltage-gated ion channels, named according to respective permeable ion: potassium, sodium, calcium and chloride channels. Ion current through the voltage operated channels represents a passive flow of ions along the electrochemical gradient defined by both transmembrane potential and ion concentrations in intracellular and extracellular space (Hille 2001). Current flowing through an ion channel solely due to electric driving force could be defined by Ohm's law (1) as:

$$
I=\frac{V-V_{R}}{R}
$$

where $\mathrm{I}$ is the current, $\mathrm{V}$ is the actual membrane potential,
$V_{R}$ is the reversal potential and $\mathrm{R}$ is the cell resistance. $V_{R}$ depends on intracellular and extracellular concentration of each permeable ion. The reversal potential for a mixture of monovalent and divalent ions may be calculated according to Goldmann-Hodgkin-Katz equation (Hille 2001) or evaluated experimentally as the membrane potential at which the amplitude of the respective ion current is zero.

The voltage operated ion channels exist in one of three functional states: closed, open or inactivated (Fig. 1A). Closed or resting state represents the channel conformation in which the channel is ready for activation, i.e., it is capable to open upon membrane depolarization. The most common depolarizing impulse used in patch clamp experiments is a rectangular voltage pulse (Fig. 1B). When the channel turns into its open or activated state the ion current passes through the channel pore and inward current increases (Fig. 1B). Prolonged depolarization of the cell membrane shifts the channel into its inactivated state. This process is reflected by relatively slow current decay (Fig. 1B). Both closed and inactivated channel states are nonconductive states. The closed channel is ready for immediate reopening, while an inactivated channel must recover from the inactivation before being able to open. Recovery from inactivation (the shift from an inactivated into a closed state) is a slow process lasting seconds to minutes. The voltage dependence of the distribution of channels among their functional states obeys Boltzmann distribution.

Current $\mathrm{I}(\mathrm{V})$ flowing through an ion channel at specific membrane potential $\mathrm{V}$ is proportional to both driving force and opening probability. Figure 2 demonstrates two theoretical situations: Figure 2A shows a current carried by an ion with positive reversal potential, Figure 2B illustrates current carried by an ion with negative reversal potential. While the former example describes quite closely voltage dependent sodium and calcium channels, the latter corresponds to hypothetical potassium channel lacking rectification phenomenon. 

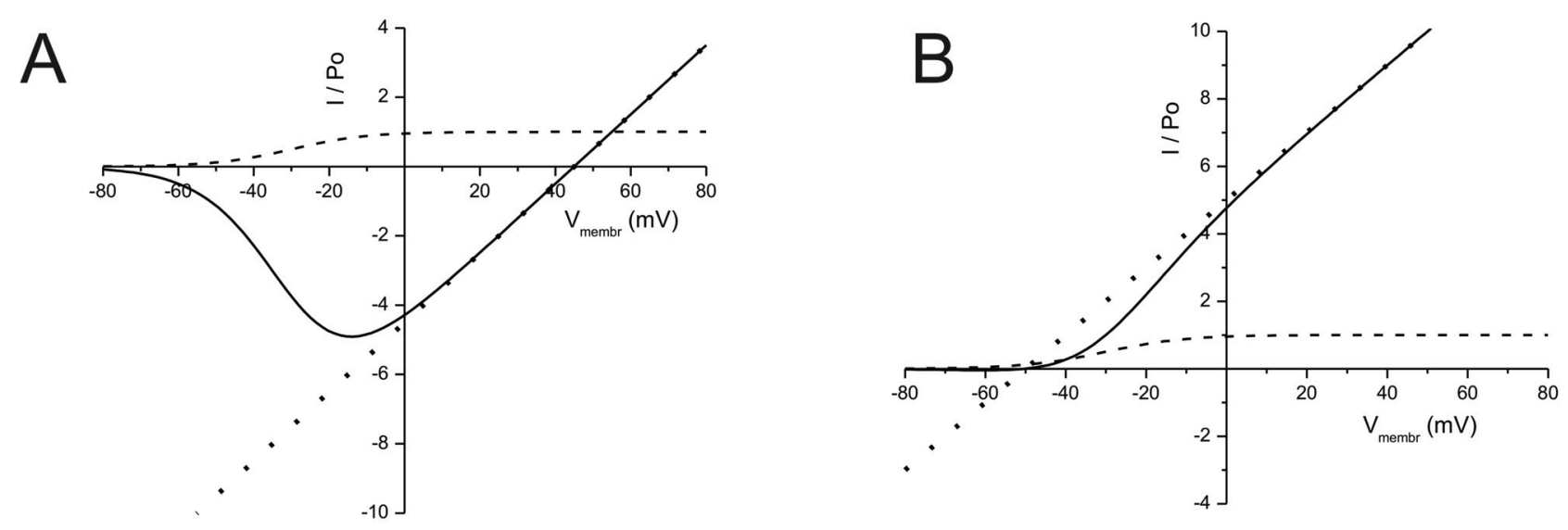

Fig. 2. Dashed lines illustrate Boltzmann-type voltage dependence of an open probability $P_{0}$ of a channel. Dotted lines demonstrate linear Ohmic driving force for a situation in which reversal potential has a positive value (A) and a negative value (B). Solid lines represent product of both relations mimicking current-voltage (I-V) relation.

\section{Measurement of individual ion currents}

\section{Freshly isolated cells}

The patch clamp method was developed for experimental analysis of ion currents in small cells freshly isolated from various organs of laboratory animals like rat, guinea pig, hamster, rabbit or frog. Such cells contain great variety of voltage-activated sodium, calcium, potassium or chloride channels. Use of bath and pipette solutions mimicking closely physiological conditions enables the measurement of action potentials under the current clamp conditions. For the purpose of measurement of specific ion current under the voltage clamp conditions this current must be pharmacologically isolated from all other currents. Permeable ions in physiological solutions can be replaced by suitable impermeable ions of the same valency. In order to suppress potassium current, $\mathrm{K}^{+}$may be replaced by $\mathrm{Cs}^{+}$ which cannot pass through most of voltage dependent potassium channels. Similarly, replacement of $\mathrm{Na}^{+}$by n-methyl-d-glucamine eliminates voltage-dependent sodium current. Chloride ions may be replaced by methylsulfonate ions.

This approach cannot be applied for calcium channels. In the absence of calcium ions these channels are permeable for any other divalent cation and in their absence may be substituted by monovalent cations. There are also other limitations. Cell viability in the experiment under the non-physiological conditions is shortened. Further, ion replacement may not fully suppress specific type of ion current, therefore it is supplemented by pharmacological blockers. Their choice is complicated by the existence of multiple subtypes of voltage-dependent ion channels.

Tetrodotoxin is a specific blocker of all known voltage-dependent sodium channels. For inhibition of great part of the potassium current tetraethylammonium can be successfully applied. All subtypes of voltagedependent calcium channels are inhibited by certain divalent cations like $\mathrm{Cd}^{2+}$ or $\mathrm{Ni}^{2+}$, nevertheless, these cations can block also other classes of voltage-dependent ion channels. Calcium channels are divided into L-type, neuronal and T-type calcium channels. L-type calcium channels are blocked by dihydropyridines (DHP), nevertheless, DHPs inhibit also some subtypes of potassium channels and may partly inhibit T-type calcium channels. Neuronal calcium channels are highly specifically inhibited by conotoxines and spidertoxines. Specific blocker of T-type calcium channels is kurtoxin. In order to inhibit fully calcium current in some cells complex cocktail of organic blockers must be used. The channel blockers are not absolutely specific and affect the cell excitability in a complex way.

Main advantage of use of freshly activated cells in experiments is preservation of their natural signaling pathways. The drawback is impossibility to investigate function and regulation of a single particular type of ion channel.

\section{Recombinant ion channels}

The end of the last century was an era of cloning. Nine representatives of voltage-dependent sodium channels (Catterall et al. 2003a), ten calcium channels (Catterall et al. 2003b) and more than one hundred potassium channels (Gutman et al. 2003) were cloned from various mammals including humans. The 
expression of recombinant ion channels in suitable system made it possible to study their properties without the necessity of pharmacological intervention. Several immortalized mammalian cell lines are used as an expression system. Most commonly used are $\mathrm{CHO}$ (Chinese hamster ovary), HEK 293 (human embryonic kidney) and tsA201 (HEK cells permanently expressing T-antigen, which promotes the replication of viral promoter-containing products). These cells do not express endogenous sodium and calcium voltage-dependent channels and therefore are appropriate for their study. On the other hand, they do express several subtypes of voltage-dependent potassium channels and thus, their application in these studies is limited (Kurejová et al. 2007). Main advantage of using recombinant channels is the possibility to study a precisely defined channel type. Further, advanced methods of molecular biology enable construction of various channel mutants in which whole segments or individual amino acids can be exchanged. Such mutants allow detailed investigation of the structure-function relation of specific ion channels and/or mapping of binding sites of pharmacological substances. The main disadvantage of the expression system is the absence of natural signaling pathways, which are present in freshly isolated organ cells.

\section{Measurement of voltage-dependent channel gating. Example of T-type calcium channel}

We shall describe the common approach to the measurement of channel activation, deactivation and inactivation on an example of T-type calcium channels expressed in HEK 293 cells.

\section{Channel activation}

To evaluate the voltage dependence of current activation a series of depolarizing steps with increasing amplitude is applied from suitable holding potential (HP; Fig. 3A). HP is a preset membrane potential, which is maintained by a patch clamp amplifier. HP should be set to a value negative enough to keep all channels in their closed or resting state. For T-type calcium channels this value is $-100 \mathrm{mV}$. Application of depolarizing pulses leads to transition of channels into an open state and to measurable inward current. Example of current traces measured from $\mathrm{Ca}_{\mathrm{V}} 3.3$ channel using the voltage protocol shown in Figure 3A is in Figure 3B. As the reversal potential for $\mathrm{Ca}^{2+}$ ions is positive, voltage dependence of current amplitudes (I-V curve) is bell-shaped (Fig. 3C). Such voltage dependence could be fitted by combined Ohm and Boltzmann relation (2)

$$
I(V)=\left(V-V_{R}\right) * \frac{G_{\max }}{1+e \frac{-\left(V-V_{0.5}\right)}{d V}}
$$

I(V) is the amplitude of the current activated by the depolarizing pulse to the membrane voltage $\mathrm{V}, \mathrm{V}_{\mathrm{R}}$ is the reversal potential, $G_{\max }$ is so-called maximal slope conductance, $\mathrm{V}_{0.5}$ is half-maximal activation voltage, i.e., membrane voltage at which current with amplitude corresponding to a $50 \%$ of maximal current amplitude can be observed and $\mathrm{dV}$ is an activation slope factor characterizing steepness of voltage dependence of current activation around the activation midpoint. Fit of experimental data in Figure $3 \mathrm{C}$ by equation 2 resulted in values $\mathrm{V}_{\mathrm{R}}=+36.6 \mathrm{mV}, \mathrm{G}_{\max }=14.2 \mathrm{nS}, \mathrm{V}_{0.5}=-46.1 \mathrm{mV}$, $\mathrm{dV}=4.5 \mathrm{mV}$.

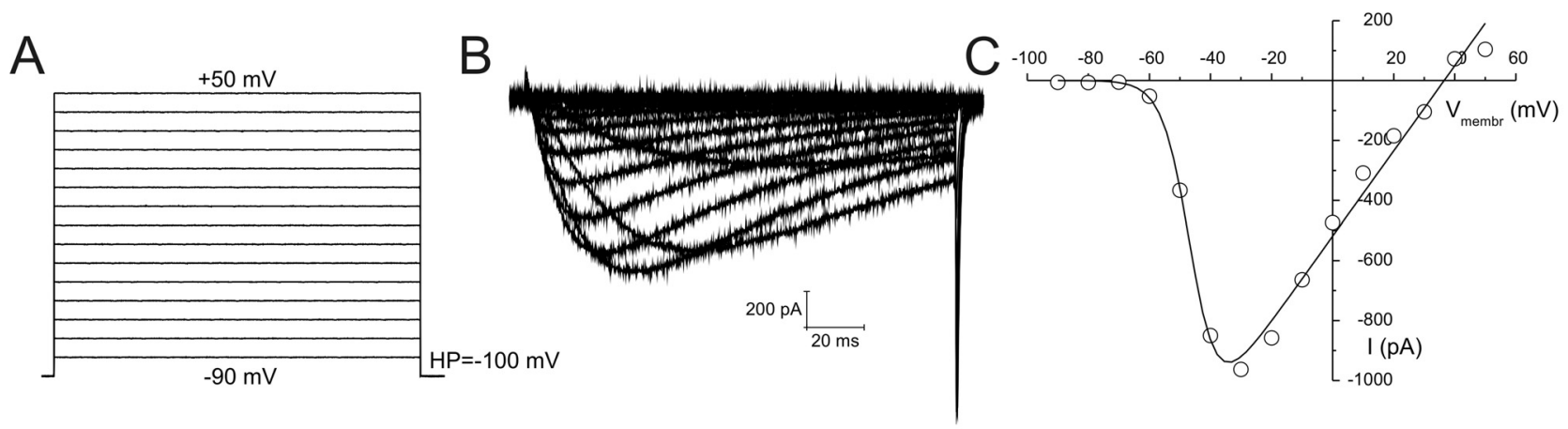

Fig. 3. Voltage protocol used for measurement of current-voltage (I-V) relations (A), corresponding current traces (B) recorded by protocol $A$ and an I-V relation constructed from amplitudes of these current traces $(\mathbf{C})$. Solid line represents fit of experimental data by the equation (2). Currents were recorded from Cav3.3 channels. 
A

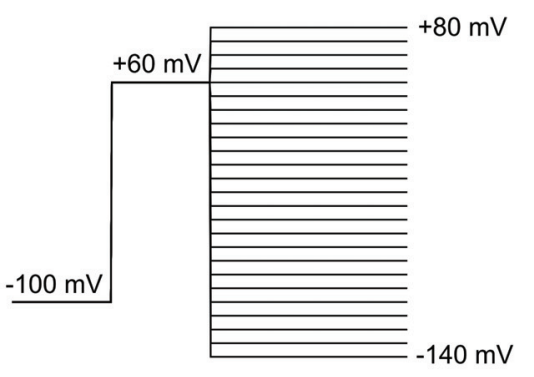

B

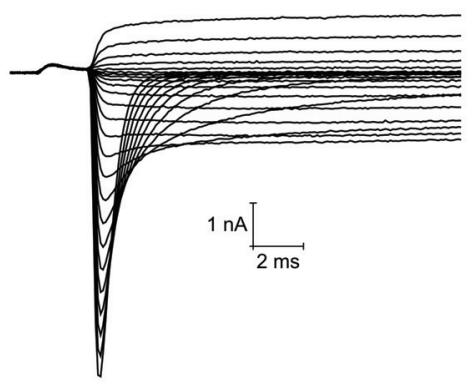

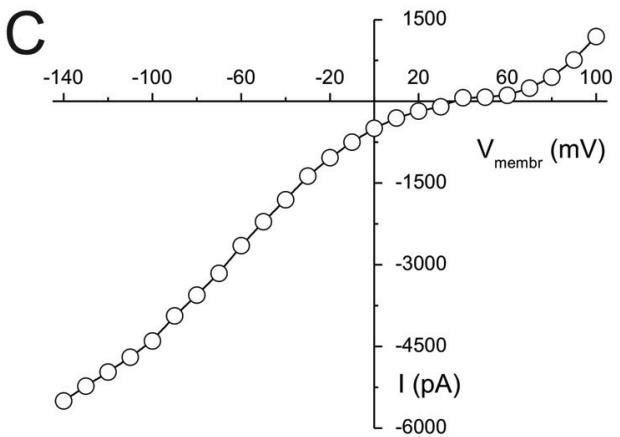

Fig. 4. Voltage protocol for the analysis of channel deactivation (A), current traces recorded using this protocol (B) and voltage dependence of tail current amplitudes evaluated from these current traces (C) - so-called instantaneous I-V relation. Currents were recorded from Cav3.3 channels.

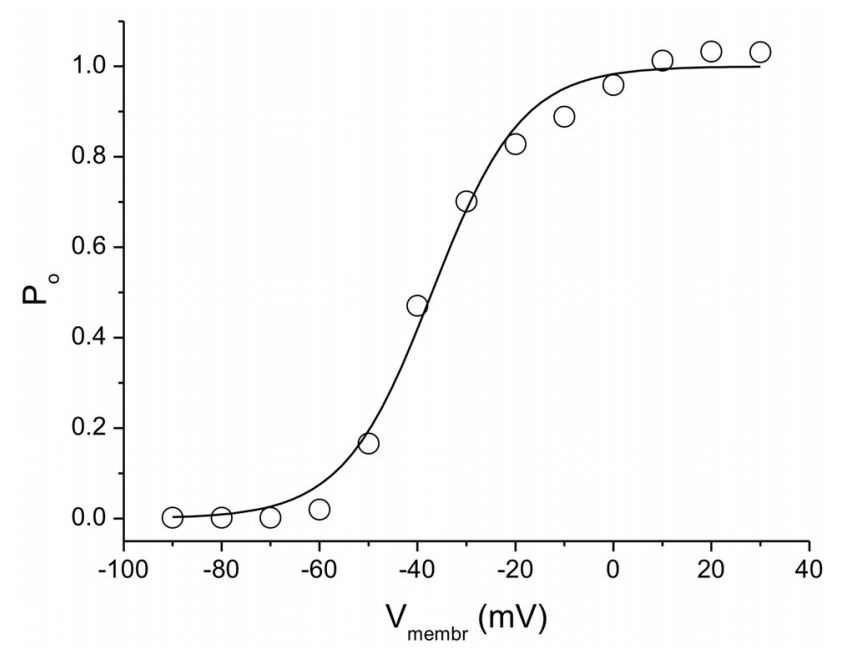

Fig. 5. Voltage dependence of channel opening probability was obtained by division of I-V data shown in Figure $3 \mathrm{C}$ by instantaneous I-V data presented in Figure 4C. Solid line represents fit of experimental data by the Boltzmann equation.

As demonstrated in Figure 3C, channel behavior in the proximity of the reversal potential deviates from the theory described by the equation (2). It should be noted that theoretical reversal potential for $\mathrm{Ca}^{2+}$ is about $+150 \mathrm{mV}$ while the observed reversal potential is about $+40 \mathrm{mV}$. This is caused by loss of ion selectivity in the outward direction in the presence of $\mathrm{Cs}^{+}$in the pipette solution. The observed outward current is carried mostly by $\mathrm{Cs}^{+}$ions.

\section{Current deactivation}

Deactivation or channel closing represents transition of the channel from open to a closed state (Fig. 1A). This transition is reflected in a so-called tail current observable at the end of the depolarizing pulse
(Fig. 1B) and is much faster than channel inactivation manifested by current decay during the depolarizing pulse. At the end of the pulse a part of the channels is still in an open state. When the membrane becomes repolarized to the HP the electrical driving force dramatically increases. As a result, the inward current amplitude increases abruptly and is followed by rapid decay reflecting the fast channel closing. The voltage dependence of the kinetics of channel deactivation is measured by a voltage protocol shown in Figure 4A. Brief depolarizing pulse to $+60 \mathrm{mV}$, i.e. to membrane potential at which channel opening probability reaches its maximum, opens virtually all available channels. This depolarization is followed by steps to repolarizing membrane potentials. With increasing repolarizing potential deactivation kinetics becomes slower (Fig. 4B).

The amplitudes of the current measured during repolarization phase may be used for construction of socalled instantaneous current-voltage curve (Fig. 4C). Since brief depolarization to $+60 \mathrm{mV}$ opens virtually all channels present in the cell, this current corresponds to a maximal current measurable at the specific repolarization potential.

\section{Opening probability of the channel}

The channel opening probability $\left(\mathrm{P}_{\mathrm{o}}\right)$ may be precisely measured from single channel recording only. Yet, there is a possibility of approximate evaluation of $\mathrm{P}_{\mathrm{o}}$ from the whole-cell patch clamp data. Current amplitude $\mathrm{I}_{\mathrm{IV}}$ measured using a conventional I-V protocol (Fig. 3A) could be defined as

$$
I_{I V}(V)=P_{0}(V) * N * i(V)
$$



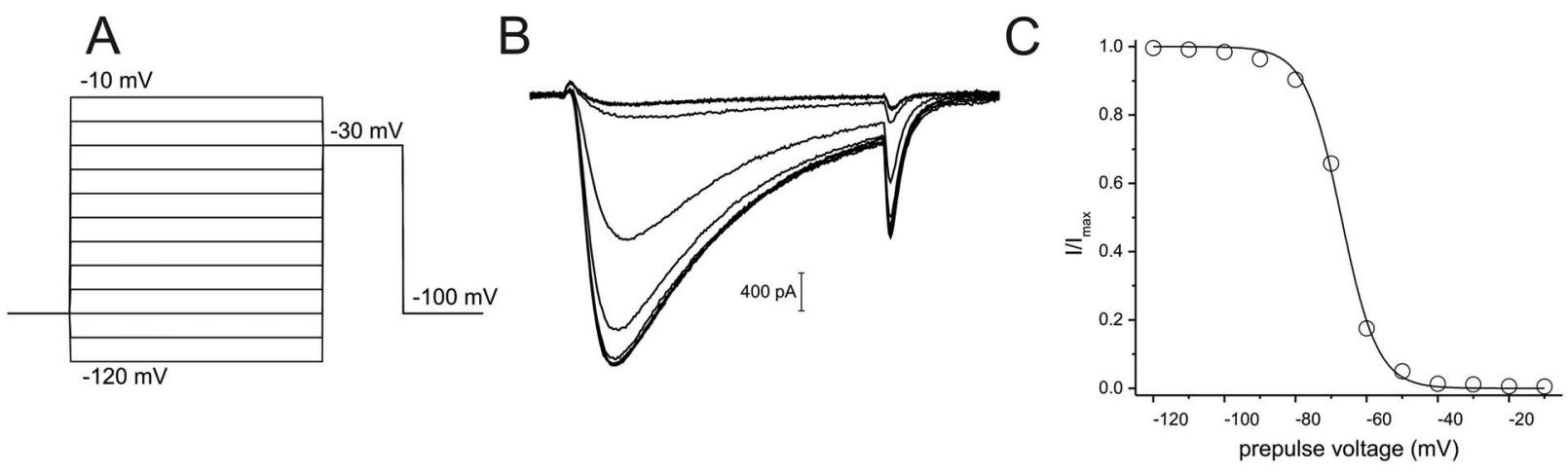

Fig. 6. Voltage protocol for recording the steady-state inactivation (A), an example of current traces recorded using this protocol (B) and voltage dependence of amplitudes of these current traces (C). Solid line represents fit of experimental data by the equation (5). Currents were recorded from Cav3.1 channels.

where I(V) represents whole cell current amplitude measured at the membrane voltage $\mathrm{V}, \mathrm{i}(\mathrm{V})$ corresponds to unitary current (i.e., amplitude of the current flowing through the single channel) measured at the same membrane potential, $\mathrm{N}$ is total number of the channel in the cell and $\mathrm{P}_{\mathrm{o}}(\mathrm{V})$ is opening probability of the channel at the membrane potential V. The same equation describes the current amplitude measured using instantaneous IV protocol (Fig. 4A), nevertheless, this protocol is designed to maximize channel open probability so it may be postulated that $\mathrm{P}_{\mathrm{o}}(+60) \approx 1$. Therefore

$$
\frac{I_{I V}(V)}{I_{\text {inst } I V}(V)}=P_{0}(V)
$$

Such relation calculated from the data demonstrated in Figures 3 and 4 is shown in the Figure 5. Boltzmann fit of these data yielded values $\mathrm{V}_{0.5 \text { act }}=-37.1$ $\mathrm{mV}$ and $\mathrm{dV}=9.1 \mathrm{mV}$. These values differ from those obtained from fitting an I-V relation in the Figure 3. The reason for this difference is that the graph in the Figure 5 shows voltage dependence of channel's opening probability i.e. reflects a single process of channel activation. Current amplitudes used for a construction of an I-V curve in the Figure 3 reflect result of two processes, voltage dependent channel activation and voltage dependent channel inactivation, which inevitably takes part during depolarizing pulse.

\section{Steady-state inactivation of the channel}

The membrane depolarization also leads to a transition of channels into an inactivated state either through an open state or directly from the closed state (Fig. 1A). Voltage dependence of current inactivation is measured by the voltage protocol shown in Figure 6A.
During the sufficiently long conditioning prepulse equilibrium (steady state) is established between closed and inactivated state. At hyperpolarized prepulse potential all channels remain in closed state. During the following test pulse a current with maximum possible amplitude is activated (Fig. 6B). The proportion of closed channels, i.e. channels available for opening during the test pulse, decreases with increasing amplitude of the prepulse. The voltage dependence of amplitudes of the current measured during the test pulse (Fig. 6C) can be fitted by the Boltzmann equation (5)

$$
\frac{I(V)}{I_{\max }}=\frac{1}{1+e \frac{V-V_{0.5}}{d V}}+I_{0}
$$

$\mathrm{I}(\mathrm{V})$ is amplitude of the current measured during the test pulse following after the conditioning prepulse with an amplitude $\mathrm{V}$ and $\mathrm{I}_{\max }$ is maximum current amplitude measured during the test pulse. $\mathrm{V}_{0.5}$ represents halfmaximum inactivation voltage, i.e., membrane voltage at which current with amplitude corresponding to a $50 \%$ of maximum current amplitude can be observed and $\mathrm{dV}$ is an inactivation slope factor characterizing steepness of voltage dependence of current inactivation at the inactivation midpoint. $\mathrm{I}_{0}$ is the value to which converges current amplitude measured during the test pulse, i.e., non-inactivating part of the current. Fit of experimental data shown in the Figure $6 \mathrm{C}$ resulted in values $\mathrm{V}_{0.5}=-67.2 \mathrm{mV}, \mathrm{dV}=5.0 \mathrm{mV}$ and $\mathrm{I}_{0}=0.01$, i.e., $1 \%$ of all channels do not inactivate even after $5 \mathrm{~s}$ long depolarization to $-10 \mathrm{mV}$.

\section{Conclusion}

The whole cell patch clamp is a powerful tool in 
the assessment of ionic currents underlying cell excitability. Voltage protocols described above belong to the most common experimental protocols used for characterization of individual ion channels and for the analysis of alterations caused by pharmacological interventions. In recent years, several automated patch clamp systems have been developed. These systems are optimized for a fast high-throughput screening of new pharmacological substances. However, the analysis of mechanisms underlying the action of drugs or detailed description of structure-function relations of individual channels requires the participation of a qualified investigator.

\section{References}

CATTERALL WA, GOLDIN AL, WAXMAN SG: International Union of Pharmacology. International Union of Pharmacology. XXXIX. Compendium of voltage-gated ion channels: sodium channels. Pharmacol Rev 55 : 575-578, 2003

CATTERALL WA, STRIESSNIG J, SNUTCH TP, PEREZ-REYES E: International Union of Pharmacology. International Union of Pharmacology. XL. Compendium of voltage-gated ion channels: calcium channels. Pharmacol Rev 55: 579-581, 2003.

GUTMAN GA, CHANDY KG, ADELMAN JP, AIYAR J, BAYLISS DA, CLAPHAM DE, COVARRIUBIAS M, DESIR GV, FURUICHI K, GANETZKY B, GARCIA ML, GRISSMER S, JAN LY, KARSCHIN A, KIM D, KUPERSCHMIDT S, KURACHI Y, LAZDUNSKI M, LESAGE F, LESTER HA, MCKINNON D, NICHOLS CG, O'KELLY I, ROBBINS J, ROBERTSON GA, RUDY B, SANGUINETTI M, SEINO S, STUEHMER W, TAMKUN MM, VANDENBERG CA, WEI A, WULFF H, WYMORE RS: International Union of Pharmacology. International Union of Pharmacology. XLI. Compendium of voltage-gated ion channels: potassium channels. Pharmacol Rev 55: 583-586, 2003.

HAMILL OP, MARTY A, NEHER E, SAKMANN B, SIGWORTH FJ: Improved patch-clamp techniques for highresolution current recording from cells and cell-free membrane patches. Pflugers Arch 391: 85-100, 1981.

HILlE B: Ion Channels of Excitable Membranes. Third Edition. Sinauer Associates, Inc. Sunderland, Massachusetts, U.S.A., 2001, 814 p.

KUREJOVÁ M, UHRÍK B, SULOVÁ Z, SEDLÁKOVÁ B, KRIŽANOVÁ O, LACINOVÁ L': Changes in ultrastructure and endogenous ionic channels activity during culture of HEK 293 cell line. Eur J Pharmacol 567: 10-18, 2007. 